\title{
Labyrinthe
}

39 | 2012 (2)

«Et si...? » La cause du contrefactuel

\section{Accident d'une fulguration qui met hors du monde}

\section{Alessandro Delcò}

\section{OpenEdition}

Journals

Édition électronique

URL : http://journals.openedition.org/labyrinthe/4282

DOI : $10.4000 /$ labyrinthe.4282

ISSN : 1950-6031

Éditeur

Hermann

Édition imprimée

Date de publication : 31 décembre 2012

Pagination : 137-151

ISBN : 9782705682637

\section{Référence électronique}

Alessandro Delcò, « Accident d'une fulguration qui met hors du monde », Labyrinthe [En ligne],

39 | 2012 (2), mis en ligne le 10 janvier 2013, consulté le 30 avril 2019. URL : http://

journals.openedition.org/labyrinthe/4282 ; DOI : 10.4000/labyrinthe.4282

Propriété intellectuelle 


\title{
Accident d'une fulguration qui met hors du monde
}

\author{
Alessandro Delcò \\ alessandro.delco@univ-paris8.fr
}

\section{Heureuse archi-tromperie}

À l'origine de la pensée il y a Eidos $^{1}$ - éclat sans substance ni sens, archi-être de pure apparence avant toute chose. Ce qui advient d'abord, c'est le pur avoir lieu sans abord, qui concentre en soi tous les prestiges du non-être. Effraction sans plus, sans dépôt, dont le seul propre est l'absence de toute propriété, le rejet de tout attribut, la récusation de toute détermination, et par voie de conséquence l'impossibilité de toute individuation. Le premier est l'ultime sur l'échelle des êtres, à savoir indigence extrême, éminente incomplétude ${ }^{2}$, saillie sans fond, surrection immédiatement déchue. Le plus intense n'est que dans le disparaître d'un éclair fugitif - tout en abîme. Là où ça brille le plus, c'est au moment d'évanouissement, à l'instant où la pointe de la singularité retombe dans l'indéfini de la nuit. N'empêche que ce comble de lumière produit les effets les plus profonds, les plus dévastateurs... Il capture comme l'hypostase la plus dense, comme un pôle irradiant absolument indéfectible il aimante. Mais en attirant à soi - lui qui n'est rien, donc nulle part dans l'espace ni dans le temps -, il met hors du monde, arrache aux coordonnées fondatrices de l'expérience, coupe de la temporalité lente des parcours empiriques. Rapt de l'intellect dans le ciel vide de l'éclatante extériorité, blessure à mort de la sensibilité par la beauté resplendissante de ce signe insigne, rejeton du néant. Le plus creux semblant est ce qui séduit maximalement en vertu de sa vacuité même, et de ce fait enclenche la chasse sans fin à l'original perdu. L'inauthentique par définition, par position et par

1. Empédocle, Fr. 40.

2. Héraclite, Fr. 65. 
nature engendre la quête éperdue de l'authentique qu'il n'a jamais été, le masque scintillant interminablement promène à sa suite la cohorte des chercheurs naïfs d'essence ${ }^{3}$. Jeu de fuites de l'apparaître. Plus le manque d'être est constitutif et irrémédiable, plus la quête du substrat substantiel est acharnée, plus la recherche d'un remède à l'effondrement principiel devient fiévreuse, plus la réparation symbolique de l'immensurable perte originaire s'impose comme tâche fondamentale. À défaillance extrême, suppléance extrême. Que la perte soit purement fantasmatique, n'importe. À l'opposé, cela explique le caractère dévorant, follement jusqu'au-boutiste de l'entreprise.

La métaphysique comme science de l'être en tant qu'être, depuis toujours à la recherche de son noyau le plus pur, de son intact primordinal, du contact avec le point-source éblouissant ${ }^{4}$, procéderait donc d'un leurre, c'est-à-dire du coup d'envoi d'un manque qui ne s'est jamais produit, sauf en imagination, et qui pourtant s'inscrit comme une évidence déchirante au plus intime de nous-mêmes. Bien que totalement fictive, l'archi-fulgurance pour nous a bien eu lieu. D'après une logique des plus paradoxales, elle a été éprouvée comme la dépossession du plus précieux, savoir comme l'irréparable proto-spoliation de la lumière. Toutefois, personne n'est à l'origine de la perte de l'Origine, car c'est l'être même, en quelque sorte, qui s'est enfui en se soustrayant à soi-même suivant la fatalité de ses propres lois, le splendide étant le plus fugace, et par suite aussi ce qui est destiné à creuser le manque le plus cruel (rien qui ne puisse manquer davantage que ce qui n'a même pas eu le temps d'être, que ce sur-être «mythique » éternellement en deçà de la barre de l'existant). Or l'abîme du manque ne pouvant rester béant sans nuire - assez vite il entraîne l'affectivité dans la déréliction ${ }^{5}-$, il appelle ce qui devra le combler d'une manière tout aussi artificielle, via une contre-fiction compensatrice de même force. La perte du Jour ne se laisse racheter que par la fabrication d'une multitude de lumières secondes, dont le scintillement nombreux doit recréer l'illusion de l'éclat premier; la fuite du mirifique mirage ne se laisse rattraper que dans la prolifération débridée des substituts. Il faut tout le bruyant carnaval des pseudo-équivalents du

3. Il se pourrait que l'aventure plurimillénaire de la philosophie commence par une séquence qui n'a pas lieu - étrange inexistant symptomal aux répercussions inépuisables.

4. Plotin, Ennéades, VI 8, 18.

5. Heidegger, Etre et temps, $\$ 40$. 
pseudo-premier, toute une surproduction de succédanés pour chasser la mélancolie engendrée par la chute du Suprême. Sa retombée en masse grise rend la pensée orpheline du fulgurant archi-archè et en même temps en fait la dure frappe, l'empreinte pathétiquement dépathétisée, la tonalité du détachement analytique. Heureuse simili-catastrophe inaugurale!

Encore qu'imaginaire, le désastre primitif est à l'origine du régime foncièrement catatonique de la pensée ${ }^{6}$, puisque le merveilleux initial est ce qui, en la paralysant, la met du même coup en mouvement, l'un n'allant pas sans l'autre, la tétanisation étant la condition d'incubation stricte, parfaite, irremplaçable, ironique... de toute suractivité noétique. Régime biphase, donc, fait conjointement de suspens et de paroxysmes, de lenteurs exaspérantes et d'accélérations subites, de surplaces et de raccourcis éblouissants, de séquences réflexives plus que monotones, où il s'agit de revenir sur les distinctions, de les rectifier, de raffiner, de dissiper tout reste d'obscurité jusque dans les moindres recoins, et d'inventions étincelantes, où l'enjeu est de saisir à toute vitesse l'ekphaines en fuite, en instance de disparition dès apparu, et dont l'évanouissement ramène l'étourdissante confusion de l'origine. De ce passage en flèche, ne reste qu'un frémissement qui menace d'emporter la faculté de comprendre dans une étrange ivresse $e^{7}$. Le trop de lumière s'effondre en tache aveugle, si bien que toutes les distinctions laborieusement acquises par voie d'analyse et de vérification, pas à pas, de proche en proche, à longueur de journée, sont brouillées par le retour soudain de cette nuit-là. Il faut tout reprendre dès le début, à nouveaux frais, comme si rien n'avait encore été pensé, comme si aucun repère n'avait pu être fixé, l'évaporation du trait se soldant par l'invasion de l'informe. D'une certaine façon, le désastre est intemporel et de chaque instant, la katastrophè des formes - qu'elles soient figures, états, types, ou genres - étant le fait même du temps, de son éternel retour ponctuellement annihilant. Autrement dit, l'accident de la fulgurance est ce qui arrive tout le temps, à chaque pointe de présent, à ceci près qu'il n'est chargé d'une portée absolument décisive que par extraordinaire, lorsqu'il est perçu en position chorismatique de pré-principe. Seulement là, il devient le premier s'offrant se dérobant, lançant la pensée et lui cassant pour ainsi dire les reins. C'est le traversant sauvage

6. Deleuze, L'anti-CEdipe, Paris, Minuit, 1972.

7. Kierkegaard, entre autres, Propos sur le mariage, in Etapes sur le chemin de la vie, trad. F. Prior et M.-H. Guignot, Paris, Gallimard, 1975, p. 112-114. 
à peine entraperçu du coin de l'œil, l'impétueux dinos hors de saisie qui tout ensemble dépeuple et féconde. Il sème le trouble dans l'enceinte paisible de la philosophie, en brise l'immémorial battement eurythmique, en met à mal les belles constantes spéculatives ${ }^{8}$, bref, réinjecte le venin de l'inquiétude dans les veines de la somnolente doctrine.

\section{Démarche intrinsèque}

Un dehors foudroyant vient bousculer intempestivement les cadres trop bien justifiés de la pensée. Il est ce qui déborde par excellence, la sur-existence inintégrable qui ne peut être d'entrée réinvestie dans le jeu des formes canoniques. En brillant l'intervalle d'un instant, il défait les contours, éparpille les limites, interrompt le cours normé des choses. Ce pur passage sans être ${ }^{9}$, cet extrinsèque au plus haut point fait pourtant incise dans le temps et l'espace, à telle enseigne qu'il projette hors d'eux, dans la région à la fois dense et éthérée des idées. En s'effaçant sur son passage même, il efface d'un trait les marques de l'entendement, les positions et les oppositions sur lesquelles il se règle, les mesures qu'il se donne et qu'il donne aux choses, afin qu'elles ne sortent pas de leur épure, i.e. de la juridiction à l'intérieur de laquelle uniquement elles peuvent faire sens. Le fulgurant indétermine les déterminations de l'analyse, perturbe les procédures de la logique, ses figures, modes et règles, et par là ouvre la brèche de l'illimité par où viennent les idées. D'un coup on passe du milieu tempéré de l'expérience, où la finitude tient lieu de critère du sens, sur son bord enflammé, où il n'y a qu'agitation polymorphe, individuations inchoatives, dispersion irrémédiable. On se retrouve excentré à la limite, sur l'intenable ligne d'horizon, là où tout bouge et se recroise, de sorte qu'une différence tranchée équivaut à l'indifférence la plus nette, et l'ailleurs est déjà sur place. Au point d'abîme, la limite d'extérieure qu'elle était, s'intériorise. C'est qu'à l'horizon le sensible s'écrase dans l'intelligible, la figure se perd dans le fond, le discursif s'imbrique dans le matériel et les essences deviennent alogiques, si bien que les nervures de l'être ne sont plus visibles que pour un œil divin, terriblement perçant, à même de faire le partage entre le néant tel quel et le néant qui porte

8. Gilson, Constantes philosophiques de l'Être, Paris, Vrin, 1983.

9. D'après Plotin, l'Un n'a pas d'essence (op. cit., VI 8, 12). 
déjà en creux la signature de l'être ${ }^{10}$. Dans l'élément de l'inconditionné et de l'aorgique, impossible d'enregistrer quoi que ce soit, impossible par conséquent de s'orienter. Sur cette ligne de crête, ça fulgure tempête disjointe crée et décrée sans arrêt. L'infrastructure secrète du monde surgit dans la vision hallucinée de l'éclair ${ }^{11}$ - moins que le temps minimalement minime d'un instant. Elle apparaît structurellement déformée dans le tremblement coupant de ce zigzag, et c'est le pays merveilleux qui précède la philosophie, son prélude de feu... Comme personne ne saurait vivre à cette hauteur - même pas le sage -, la philosophie recule d'un pas. Elle installe son «campement» juste à proximité.

La philosophie se pose à distance minime du pays empédocléen des foudres et des volcans, de telle façon qu'elle puisse recevoir la brûlure du grand feu primordial sans en être pour autant anéantie. Capter dans l'opérativité de l'idée l'intensité insoutenable de ce rayonnement, telle une supraterrestre énergie d'activation - voilà l'une de ses plus belles ruses. S'exposer à cette lumière, tenter de l'emprisonner dans les rets de la langue transgénérique du désaffect, où toutes les valeurs sont des variables ouvertes. Travail de transduction, de transmutation même, tellement cela tient du réservé. En dehors du réglage conforme de toute maxime - au flair il se fait, si jamais ${ }^{12}$. C'est d'ailleurs pourquoi il y aura toujours dans le travail du philosophe une petite part d'arcane qui s'accompagne d'un risque majeur, savoir le risque que ça ne se fasse pas, que ça demeure dans les limbes prestigieux de l'irréalisé. On ne peut jamais exclure l'hypothèse que ça reste du côté du non désocculté, c'est-à-dire de l'indéveloppement initial tout enroulé en soi, insistant pour toujours dans l'autoréférence, trop épris de lui-même, en un sens, pour pouvoir s'extérioriser. La radiance du fulgurant ne passe dans l'élément du spéculatif qu'à condition de rencontrer un «corps-milieu » capable de la réfracter de la bonne façon. Sans cela, elle ne peut subir la transformation métamorphique adéquate, celle qui trouve le moyen de transposer le météorique le plus instable dans un minimum de teneur substantielle. Or, cette configuration à même d'inclure des lignes labiles à haute tension

10. D'une certaine manière, on retrouve ici la distinction kantienne entre nihil negativum et nihil privativum. Cf. Critique de la raison pure, A 292.

11. Héraclite, Fr. 64.

12. Nietzsche, Le cas Wagner, in Euvres complètes, t. VIII, Paris, Gallimard, 1974, p. 45.

Travail proprement insensé, si le sens est - comme a pu le poser Lévinas - le préajustement du dedans et du dehors. Cf. De l'existence à l'existant, Paris, Vrin (édition de poche), 1990, p. 74. 
n'est autre que l'idée, l'idée où une fois de plus converge l'extrême de la concentration impliquant l'émission de traits et de rapports. C'est parce qu'il y a toujours dans l'idée une région d'elle-même parfaitement opaque et inexplorée, en dépit de son exposition, qu'elle entre en connexion avec d'autres idées, plus exactement avec des aspects d'elles également restés à l'état indéveloppé.

Consistances paradoxales que les idées, dans la mesure où ce sont des noyaux centralement apathiques, encore qu'ils renferment toute l'excitation en provenance de l'éblouissement archétypal. Sous cet angle, elles sont à même de s'enchaîner dans la tonalité neutre caractéristique de la philosophie ${ }^{13}$. Si elles impliquent une quelconque griserie, ce sera nécessairement la grise griserie de la concatenatio des formes notionnelles, soumise à l'inspectorat implacable de la logique. Au niveau du philosophique proprement dit, c'est-à-dire de l'articulation ordonnée des idées dans la structure on ne peut plus surveillée de l'argument, la tension a déjà beaucoup baissé, étant donné qu'elle a été convertie dans la linéarité stricte du discours, où les significations sont obligées de se suivre les unes les autres, en file indienne..., sans imprévus. Il se trouve que l'idée ici bâtit son propre monde, remarquablement indifférent au monde ordinaire, et cette réalité à part forme le champ illimité des expérimentations de la pensée pure, laquelle peut évoluer sans fin dans son plan. Ce qui veut dire qu'un tel processus s'autonomise très vite, devenant par là susceptible de se réfléchir soi-même à perte de vue. Or l'univers du théorétique est si intrinsèque qu'en principe il persiste même au cas où le monde devait s'effondrer ${ }^{14}$. Même une telle éventualité ne le déferait pas, car une fois de plus il tient et se déploie entièrement en lui-même - opérativité absolue absolument souveraine. Il est sa propre loi et constitue en immanence son propre cheminement méthodique. D'où ce corollaire : l'idéalité ne chute pas avec l'empirique contingent. Elle n'est plus touchée pas l'inconstance fâcheuse de ses péripéties; elle vit désormais d'une vie séparée, inviolable, le propre de la puissance de l'éidétique étant de préserver des aléas de l'accidentalité. C'est le règne de la spéculation, où l'idéel a su conquérir le statut d'incorruptible, que

13. Husserl, Idées pour une phénoménologie pure et pour une philosophie phénoménologique, I, § 31 . 14. Schopenhauer, Le monde comme volonté et comme représentation, trad. A. Burdeau (revue et corrigée par R. Roos), Paris, PUF, 2009, § 52, p. 329. 
ce soit sous la forme d'une essence qu'aucun cas ne peut plus entamer ou bien sous la forme d'un acquis rationnel définitif ${ }^{15}$.

\section{Problématique non-positivité}

Se déroulant dans la sphère des idées - sphère d'un genre tout à fait particulier -, la philosophie n'est pas vraiment une activité de ce monde. Le fait est qu'elle souffre d'un idéalisme fondamental et incurable ${ }^{16} \ldots$, qu'elle partage en vérité avec d'autres disciplines théorétiques, les mathématiques en premier. Sa plus belle prérogative - la vie à la fine pointe de l'abstraction, voire dans les liens de l'esprit - est en même temps ce qui en marque la faiblesse, à savoir son peu d'enracinement dans la dimension hétéroclite, intimement décousue et caduque du terrestre. Comme elle trouve son site opératoire à l'horizon, elle a lieu invariablement là où les choses fuient et tendent à s'estomper. Toute en pointillé, s'avançant d'un pas ailé, la philosophie est plus en affinité avec les ombres et les scintillements - variantes notables du non-être - qu'avec les lourdes assises de l'ousia. Née du charme d'Eidos, qui déporte au-delà des concrétudes finies, elle vit sous la menace permanente de s'évanouir. Plus en phase avec le côté spectral des choses qu'avec leur côté charnel, plus proche de la matrice schématique des corps que de leur masse, plus en accointance, de par sa langue chiffrée, avec la fibre des étants qu'avec leur épaisseur, si elle connaît le privilège de pouvoir dilater l'expérience à l'extrême, c'est au prix d'une perte radicale en variété, d'un déficit chronique en contenu. Son orient - on l'a dit -, c'est le visage paradoxal de l'Idée, d'une splendeur incomparable - anti-phénomène s'il en est ! Tout bien considéré, le malheur de la pensée est d'avoir été, ne serait-ce qu'une seule fois, l'espace d'un instant fatal, maximalement sensible à l'attrait de cet insensible, qui l'a définitivement ravie dans le royaume des formes sans forme ${ }^{17}$ et des symboles miroitants tenant lieu de positivités mondaines, avec ceci pour comble, qu'ils sont même mieux qu'elles, étant entendu que les substituts inexistants des choses ne périssent pas,

15. Aristote, Seconds analytiques, I, 2.

16. Whitehead, Aventures d'idées, trad. J.-M. Breuvart et A. Parmentier, Paris, Cerf, 1993.

17. De façon tout à fait insolite en contexte grec, Plotin affirme que l'Un est sans forme (op. cit., V $5,6$ et VI 7,33$)$. 
à la différence des choses en chair et en os. Le «en creux» de la chose, son mannequin disséqué (sur lequel tester toutes ses variations, jusqu'à l'impossible), plus intéressant que la chose; son image diaphane (ayant même surface qu'elle), plus attrayante que sa présence réelle, toujours lestée d'imperfections; sa notation diagrammatique, n'en retenant que l'allure dans l'approximation anexacte du style, plus instructive que la description par le menu, illisible, de ses particularités.

Comme elle se déploie sur le plan de l'idée - instance rayonnante toute proche de la variabilité du simulacre ! -, il va de soi que la philosophie est d'essence fragile. D'une certaine façon, elle se borne à répercuter dans la lumière seconde de l'intellection le foudroiement qui l'a fait naître avant elle, dans un hors d'elle-même qu'elle ne pourra jamais résorber en totalité ${ }^{18}$. C'est qu'elle vient d'un éclat ne pouvant donner lieu qu'à un faisceau de déterminations esquissées, anticipant sur elles-mêmes, jamais en état de s'établir par voie d'auto-validation, de sorte que l'Idée correspondrait au lancer de cette conjecture première et l'intellection à la réeffectuation réfléchie, nécessairement décalée, légèrement posante, de ce jaillissement. Depuis le point le plus brillant - mens momentanea sans exemple ni suite -, de manière forcément précaire, surgit la flèche de l'idée, aussitôt que le fulgurant s'efface, pour s'effacer à son tour sous très peu ${ }^{19}$, non sans avoir toutefois tracé sa trajectoire. Mais si l'idée dans son surgir même contient le disparaître du plus éclatant, elle ne pourra jamais être de façon plénière, elle sera en somme toujours à cheval sur au moins deux modes - être transitionnel à la lisière de son oblitération. Habitée par ce discord d'origine, elle n'advient en définitive que dans son à côté - non pas dans la précision de l'univoque, mais dans la perfection illocalisable de son aura.

Tout cela se passe avant l'ordre positif du positionnel. Le pur travail de l'idée en chemin, c'est elle ne se sachant pas encore comme telle, n'ayant pas encore été corrompue par sa nomination. Plénitude étrange de cette idée aux mille facettes, axes, inflexions, n'ayant pas encore été « assignée à résidence » par le motif, échappant encore pendant un bref sursis à la misère de l'idée générale et à son caractère typé; problématique nonpositivité également, qui l'expose à la dissolution dans le sans limites. Le

18. Toujours d'après Plotin, l'Un n'a aucun rapport avec les choses, étant au-delà de tous les genres (op. cit., VI 2, 3 et VI 8,8).

19. Le texte des Ennéades (VI 5,6) dit à la lettre «kata brachù ». 
fait est que dans l'Ur-idée pulse la vie formidable de l'apeiron - milieu mouvant, immensité cruelle traçant sans cesse, quitte à faire disparaître d'un même mouvement toute trace ${ }^{20}$, à l'origine tant de la genèse des formes que de leur dégradation en indications fuyantes. Remontant du fond, l'inidentifié défait toute identité, position, installation, avancée. Se perd alors jusqu'au souvenir de la voie. Le sillage de l'idée se referme; se reforme l'uni de l'océan premier toujours égal à lui-même, platitude sans disparités, sans traces de litiges, sans rides ni lignes de force permettant de se repérer dans le quelque part le plus quelconque. Bien que toujours déjà prise dans l'archi-élémentaire enveloppant, l'idée ne vit donc qu'en exil, c'est-à-dire qu'en état d'altérité permanente vis-à-vis d'elle-même. Être biface, tenant ensemble de l'éclat et du substrat, elle puise à la générativité chaotique de l'interminé ensemble son essor différençiant et son minage, tout comme si elle ne pouvait qu'exister à la frontière, ou pour mieux dire, dans le passage des frontières - éternelle transfuge. Dès lors, rien d'étonnant qu'elle ne trouve sa consistance que dans la plasticité de l'inchoatif toujours à recommencer, qu'elle ne puisse se conjuguer en définitive qu'à l'infinitif.

C'est là qu'apparaît sa parenté intestine avec le temps (qu'elle voudrait pourtant court-circuiter). En effet, à l'instar du temps, elle existe à peine, dans la puissance électrique de l'instant qui court immanquablement à sa perte, condition de son renouveau. Être sans subsistance en stricte logique, qui est tout de brasillement, essence impensablement inessentielle, sa nature n'est pas sans rappeler le statut outrancièrement problématique de l'être en puissance, si dramatiquement privé d'un ubi consistam. Entre ce qui n'a encore aucune effectivité - tout entier replié dans son soi en deçà de toute manifestation - et ce qui a déjà trouvé son plein déploiement dans l'affirmation de l'être, qu'y a-t-il ? Qu'y a-t-il dans cet interstice étrange, qu'on ne sait si trop vide ou trop plein, trop calme ou trop hérissé de crises ? Quelque chose peut-il seulement se tenir là, à cet endroit intenable, s'arrêter sur l'arête de cette limite glissante, au point d'indistinction où bifurque tout le temps, invisiblement, la lame du temps ${ }^{21}$ ? Cependant,

20. Sous ce rapport, il est l'analogue de ce que Freud appelle «processus primaire». Cf.L'interprétation des rêves, $7, \mathrm{E}$.

Parmi les philosophes contemporains (abstraction faite de Heidegger), Lacoue-Labarthe est probablement celui qui a eu le sens le plus aigu du Terrible. Cf. par exemple Préface à La disparition, Paris, Christian Bourgois, 2009.

21. Lacoue-Labarthe, op. cit., p. 46. Pour un équivalent dans le temps musical, cf. Lévinas, op. cit.,p. 46. 
l'être n'est qu'en raison de cet événement diagonal ontologiquement nul. Il n'est qu'en vertu d'une obliquité qui en traversant déplace sans arrêt le point d'arrêt - l'entêtement de l'instant qui ne veut pas mourir, céder la place, s'en aller. Le fulgurant doit donc fulgurer l'instant, le mettre à mort - autrement, pas d'instant d'après ${ }^{22}$. Moment vertigineux de l'ekstasis, où le temps repasse pour ainsi dire à zéro, par le point à la fois annihilant et irradiant de la source, afin de s'y ressourcer. Ce n'est que dans la perte radicale du sens - à l'occasion de chaque instant - que le sens - du temps, de l'être - peut fulgurer. L'« en puissance» est le moment d'un croisement effroyable entre ce qui chute dans le néant et ce qui monte à l'être - le même -, c'est le chiasme instantané, terrifiant, entre l'être qui passe pour toujours à la trappe et celui qui vient le remplacer en toute hâte, le tour d'un présent.

Dans son exercer le plus exigeant, la philosophie opère dans cet élément de la transposition, davantage, de la transpassibilité pure ${ }^{23}$, où tout s'inscrit et rien ne peut s'inscrire durablement, puisqu'une espèce d'immobile mobilité dévore aussitôt chaque tentative d'«impression». Toute la difficulté pour elle est alors d'arriver à fixer quelques repères, premiers jalons d'un parcours possible. Mais pour ce faire, elle doit trouver la disposition libérant l'affect d'axiome, c'est-à-dire le geste noétique consistant à placer une balise en plein vide. Or, le signe le plus ténu peut suffire à casser la monotonie d'une surface se poursuivant interminablement toujours pareille, étale de part en part - inlassable répétition à l'identique de l'identique. Surface habitée par une inertie corrosive de surcroît, qui interdit aux accidents d'y laisser des traces, qui distord les formes avant qu'elles n'aient pu se formuler. Il se trouve que la strate de l'Urstoff est très peuplée d'individuations à l'état naissant, destinées la plupart du temps à une carrière plus qu'éphémère. À peine surgies, même pas delinées, qu'elles se font déjà rattraper par le magma dispersant et activement «panurgique» du fond. De toute évidence, afin qu'une silhouette puisse émerger, voire une signification claire puisse prendre forme, il faut la scansion d'un déterminant qui découpe et délimite d'une main de fer. Le quelque chose naît d'une segmentation du continuum primitif à teneur excessivement uniforme, segmentation engendrant des unités de sens, voire des morphologies bien reconnaissables qui font pièce un

22. Kierkegaard, op. cit., p. 140.

23. H. Maldiney, Penser l'homme et la folie, Grenoble, Millon, 1997, p. 114. 
instant à la puissance dissolvante du fond. Mais en l'absence de toute pierre de touche et de tout modèle, au commencement ceci ne peut se produire qu'à l'aveugle. Une forme se lance, on lance une forme. Sera-telle viable ? Qui sait? Reste que du sens en général n'est possible que si la concentration folle de l'Un se distend dans le cours lié des phrases, ou dans la distribution rythmée des formes ${ }^{24}$. D'une complicatio où tout est si serré qu'il n'y a de place pour quoi que ce soit, on doit pouvoir passer à une explicatio où de l'étendue se crée, où dans l'acte de déplier quelque chose vient à l'être, quitte à redisparaître dans le mouvement qui s'ensuit.

Dans sa mouture originaire, encore toute près de sa source radicale, la philosophie peine à se déposer dans des formes stables. Elle est encore bien en deçà de la fermeté des concepts, pour ne pas dire de l'assurance quelque peu factice des grands systèmes. Toute proche de l'inquiétante intrication des racines ${ }^{25}$, adossée encore aux productions mêlées des commencements, elle a du mal à s'extraire de la dense atmosphère des origines, où une lumière trop forte fait naître génies et autres créatures fantastiques se baladant dans les airs... Là, elle est encore en contact ésotérique permanent avec le divin et ses intermédiaires, et comme les mots sont les choses, un sentiment de toute-puissance s'empare d'elle (alternant, il est vrai, avec une extrême vulnérabilité). Faute d'une méthode constituante, elle expérimente dans tous les sens. Sa richesse est dans la pluralité des directions qu'elle ose emprunter en même temps, sans trop se soucier de cohérence, le vaguement ajusté lui suffisant. L'amplitude d'abord, le souci du détail ensuite. À ce stade, elle préfère de loin le raccourci à la démonstration procurant l'explicitation complète des chaînes de raisons. C'est qu'elle estime plus élégant de procéder par allusions que de tout prouver. Bien entendu, la consistance logique stricto sensu n'est pas son fort, encore moins le maniement de la langue propositionnelle, cette langue qui ne se contente pas d'indiquer mais veut graver le fluant dans le marbre des thèses inébranlables. Toutefois, ce qu'elle perd en précision, elle le gagne en souplesse, le défaut de netteté se renversant dans la vertu de l'ouvert. Et si elle manque un peu de rigueur au sens scolaire du terme, cette pensée qui ne craint pas l'exubérance du germi-

24. Lyotard, Discours, Figure, Paris, Klincksieck, 1971, p. 283-284.

25. Schelling, Recherches philosophiques sur l'essence de la liberté humaine, in La liberté humaine et controverses avec Eschenmayer, trad. B. Gilson, Paris, Vrin, 1988, p. 201 sq., de même que Les âges du monde, trad. P. David, Paris, PUF, 1992, particulièrement le livre premier (1811). 
natif évite néanmoins le travers de la rigidité dogmatique. Bref, elle s'investit tout entière dans des montages temporaires qu'elle réinvente dès qu'ils se fatiguent.

\section{Rapports tumultueux avec le dehors}

Si malgré ces remodelages continus (ou grâce à eux) la philosophie est un processus qui se sustente entièrement lui-même - immanence d'opérations s'enrichissant indéfiniment du fait de leur inclusion mutuelle -, on pourrait être tenté d'en conclure qu'elle configure une sphère parfaitement autonome. Il semblerait par conséquent qu'elle ait son lieu atopique dans l'absolu de l'abstraction de l'idée, faisant monde pour et par soi-même. Sauf que rien n'est moins sûr, puisque l'abstraction ou la maximalisation de l'idée - passage à la limite, élévation au degré suprême - n'est concevable en fin de compte qu'à partir du socle prosaïque de l'expérience ${ }^{26}$. C'est depuis la couche basique des concrétions singulières - allant jusqu'à l'effrangé indescriptible d'une tache-, depuis le désordre presque «babelesque» des cas empiriques que le cristal de l'idée peut se former et briller d'une lumière d'autant plus vive qu'elle s'élève sur un fond sombre, emmêlé, divers, irrationnel. Le vecteur de l'abstraction prend appui, plus encore, s'enfonce dans telle série triviale, quitte à la balayer à toute allure pour en sortir à l'autre bout - tête qui se détache du reste du corps, caput où la variation vient s'éteindre, ne pouvant cependant exister en dehors du spectre des termes qu'il excède. La splendeur de l'idéal hors série demeure structurellement tributaire de l'obscure et obtuse matière, de ses racines terreuses, se ramifiant telles des tentacules voraces jusque dans l'indéfini des terminaisons les plus évanescentes ${ }^{27}$, ou à l'opposé s'entrelaçant pour former la consistance pâteuse, imprédicable, de la boue. Par un étrange télescopage, la singularité incatégorisable de l'Idée - trop en excès vers le haut - communique de manière souterraine avec l'infime degré de la matière - tout aussi insubsumable, puisque trop pluralisé vers le bas.

26. Platon, République, 510d-511c.

27. Plotin, op. cit., VI 7,33, et sur sa lancée, tout près de nous, Stanislas Breton, Matière et dispersion, Grenoble, Millon, 1993, notamment le chapitre VI et les importantes indications de méthode de la préface. 28. Platon, Parménide, 132b-134a. 
Il va sans dire qu'une discipline qui prétend former un univers à elle toute seule, un tout paradoxal ouvert uniquement sur son dedans, dont la loi d'organisation émane de lui et de lui seulement, autarcique et autotélique à la fois, ne peut avoir que des rapports compliqués avec un dehors qu'elle réduit à la portion congrue, voire qu'elle voudrait pouvoir décompter tout court. Du moment qu'elle postule son autosuffisance, ce reste pour elle ne peut être que synonyme d'existant qui dérange. Autrement dit, il n'est qu'ajout encombrant, accessoire sans fonction, chose qui alourdit sans rendre service, en un mot, superfétation. Par suite, les relations entre les ordres foncièrement disparates de l'idéel et du réel - qu'il se décline sous la forme du matériel, du sensible, du perceptif, de l'affectif, ou d'une autre manière encore - ne sont jamais réglées ${ }^{28}$. Entre eux il y aura hiatus à jamais, si bien que le passage ne pourra se faire que par un saut ${ }^{29}$ - moment de vertige immaîtrisé, sorte de réplique parodique après coup de l'instant fulgurant inaugural. Entre l'unité invincible de l'idée et la variété irréductible des choses - individus, exemples, cas et circonstances, avec en plus tout le cortège de leurs espèces mitoyennes structurellement mal définies -, la coexistence sera toujours tendue. La pure, lumineuse et hiératique Idée ne pourra jamais pactiser avec la dissémination impudique du sensible, pullulant de germes, scories, résidus et agrégats de toutes sortes, proliférant par-dessus le marché avec une vivacité stupéfiante - allergie qui s'explique par ceci, que la perfection de la forme est particulièrement affectable par l'informe, en constitue même la proie prédestinée, s'il est vrai qu'en un tournemain il l'envahit et la corrompt jusqu'aux moëlles. Raison de plus pour l'Idée de se tenir à distance d'un foyer si ostensiblement contagieux. Sauf que cela mène tout droit à la mauvaise alternative suivante : ou bien c'est l'idéel qui a le dessus sur la diversité des concrétions et expressions matérielles, mais alors la richesse bariolée du monde est perdue, puisque ramenée de force à son abrégé schématique le plus pauvre, via la capture du concept; ou bien c'est l'inverse qui se produit, le sensible l'emporte, mais dans ce cas c'est l'idée qui en pâtit, dans la mesure où elle risque de se dissoudre dans la promiscuité pandémique des constituants élémentaires du monde.

Tout le problème est de comprendre comment l'idée - matrice et symbole de la philosophie - peut avoir affaire avec son autre - distributions

28. Platon, Parménide, 132b - 134a.

29. Kierkegaard, passim. 
spatiales, séquences temporelles, extériorité de la matière, fluctuations psychiques, etc. - sans perdre sa nature propre. Il s'agit en somme de trouver le biais - nécessairement contourné, sinon labyrinthique - par lequel l'idée pourrait «s'incarner» sans rien perdre de son corps incorporel à elle, pourrait «descendre» dans la matérialité du sensible tout en restant parfaitement elle-même, c'est-à-dire intégralement idéale ${ }^{30}$ - authentique gageure, l'inclusion dans l'existant entraînant ex necessitate pour l'idéal la fin de son statut séparé, savoir la corruption de son «en tant que tel», et vice-versa l'assomption du sensible dans l'« inséité» absolument inétendue de l'idée impliquant pour lui la révocation de sa propriété cardinale, i.e. le fait de s'étaler, se distendre, se diffuser indéfiniment - toujours plus en dehors de lui-même ${ }^{31}$, au point de perdre jusqu'au souvenir de l'unité dont il a dû procéder une fois, au principe. Aut aut sans remède, si on donne toute sa portée au moment disjonctif qui fait que la philosophie entretient dès sa fondation des rapports problématiques avec le monde. Ne pouvant être en prise directe sur lui sans risquer de sombrer, elle se voit obligée de choisir dès le début une posture de retrait. Ce qui veut dire qu'elle ne peut avoir rapport à l'existence et à tout ce qui est du même ordre - corporéité, historicité, liberté etc. - que de manière biaisée. N'étant pas de taille à subir de plein fouet la violence du monde, elle la latéralise, en diffracte l'impact, l'atténue dans le feuilleté des strates de l'analyse, pour qu'elle devienne enfin assimilable dans le registre du notionnel. Sous peine de devenir aride, pire, de se réduire à un petit jeu insignifiant, elle doit malgré tout laisser entrer dans son champ au moins l'écho des grandes batailles réelles.

Une attitude de ce genre ne va pas sans ambiguïté, car ce qui prime ici n'est pas le franc accueil de ce qui se présente, mais une stratégie visant à laisser venir seulement après avoir mis au préalable suffisamment à distance ${ }^{32}$. Hospitalité sous condition, réception précautionneuse, non-hospitalité de fait, puisque ordonnée à une pointilleuse sécurisation transcendantale. Cela peut se dire dans le jargon philosophique «secondarisation des événements »-drames en tout genre -, afin d'en désamorcer la charge explosive, le but étant le report du $d a$ brutal de l'existence

30. Cela n'arrive qu'à titre tout à fait extraordinaire dans l'oeuvre d'art, comme l'avait déjà parfaitement vu Kant. Cf. Critique de la faculté de juger, $\$ 49$.

31. Descartes, Méditations métaphysiques, VI.

32. Lévinas a une formule magnifique pour cela : «possession les mains libres» (op. cit., p. 72). 
sur le plan feutré du théorique. Prudence quelque peu médiocre, sinon foncièrement louche... La pensée louche pour éviter le face-à-face avec ce qui est, pour ne pas voir ce qu'il en est effectivement de l'être, pour ne pas succomber sous son poids ? Quoi qu'il en soit, on sent bien qu'elle ne fera jamais vraiment face, qu'elle ne s'affrontera pas à la pointe du péril, et c'est toute la différence, notamment, avec l'audace folle du héros tragique. On la sent rétive, à tel point que même là où elle s'expose le plus aux puissances démembrantes elle est encore en position d'observateur, non participante, retranchée derrière de hautes murailles... À l'abri, donc, sous la voûte de l'essence, et son patronage - garantie suprême que ça ne s'écroulera pas, que ça ne se dispersera pas jusqu'au bout, quoi qu'il arrive. La dissipation s'arrête in extremis. Elle butte sur une borne indestructible. Eidos - noyau quintessentiel - résiste. Tout est perdu, sauf ce qui sauve ultimement. En dépit des apparences, il ne s'est rien passé. 